\title{
Non-contact, non-obtrusive electrocardiography in clinical environements
}

\author{
Bhavin Chamadiya \\ Research \& Development \\ Daimler AG \\ Boeblingen, Germany \\ bhavin.chamadiya@daimler.com
}

\author{
Kunal Mankodiya \\ Dept. of Rehabilitation Science \& Tech. \\ University of Pittsburgh \\ Pittsburgh, USA \\ kmm197@pitt.edu
}

\author{
Manfred Wagner \\ Research \& Development \\ Daimler AG \\ Boeblingen, Germany \\ manfred.mw.wagner@daimler.com
}

\author{
Rayan Bou Nasreddine \\ Institute for Signal Processing \\ University of Luebeck \\ Luebeck, Germany \\ nasreddine@isip.uni-luebeck.de
}

\author{
Ulrich G. Hofmann \\ Institute for Signal Processing \\ University of Luebeck \\ Luebeck, Germany \\ hofmann@isip.uni-luebeck.de
}

\begin{abstract}
Even though it might be beneficial to have continuous baseline data upfront, nowadays hospitalized patients are put on a vital signs monitor not earlier then they become bed-ridden. Aim of this study is to show the feasibility of a broader monitoring concept embracing many more subjects by monitoring contact-free vital signs with sensors embedded in clinical fitments. This work demonstrates a way to measure ECG unobtrusively by capacitive coupling in various clinical settings like hospital beds, stretcher $s$ and wheel chairs. Contact ECG and non-contact ECG measurements by shielded textile electrodes are contrasted to each other and display the potential of contact-free monitoring.
\end{abstract}

Non-contact Electrocardiography, Clinical environments, textile capacitive electrodes, patient's freedom of movement, future hospital.

\section{INTRODUCTION}

In nowadays budget driven hospital environments only the most needy patients are continuously supervised by alarm monitors. So, patients on the intensive care units are clearly in need for monitoring, whereas pre-op and mobile patients aren't, even though it might provide baseline recordings useful for further diagnosis and treatment.

Besides budgetary restrictions obtrusiveness is another hindrance to permanent monitoring: No mobile patient is happy to push a cart with monitors along his way, just to maintain the optimal position of cable bound ECG electrodes. Even when using a portable Holter-type device, electrode-skin contact still forms an interface prone to degradation of conductive gel, motion artifacts and skin irritations. Metal electrodes in long term recordings might even lead to allergies and skin irritations and may result in pressure necroses.

We thus considered as an important step towards nonobtrusiveness the capacitive recording of electrical biosignals by devices not in intimate skin contact. The first capacitive recording of an ECG signal without conductive body contact was described by Richardson [1]. The surfaces of his capacitive electrodes are electrically insulated and remained stable for long-term monitoring.

Another aspect of unobtrusiveness is the integration of sensor systems in everyday clinical objects, thus avoiding any type stirrup for "wiring" a patient and still providing monitoring options.

In 2007, Y.G. Lim et al presented a study on non-contact ECG measurement on a bed during sleep [2]. They implemented an array of 8 copper clad capacitive PCB electrodes $\left(4 \mathrm{X} 4 \mathrm{~cm}^{2}\right)$ with embedded electronics and a large conductive textile electrode used as ground plane. To measure heart rate, R-peak was detected from one of the 8 channels, sorting by its sufficient quality.

In the same year, Ueno et al demonstrated influence of various factor like cloth thickness, electrode area and coupling pressure on the result of the Capacitive ECG [3]. During the measurement in the bed, all the factors had minor to major influence on the ECG signal, in that electrode area had greater impact than cloth thickness and the pressure. It was also showed here that input capacitance of the preamplifier plays dominant role in the signal coupling and should be reduces as much as possible to achieve better signal quality.

Next year, Wu \& Zhang designed capacitive electrodes with stretch conductive fabrics and was integrated beneath a bed sheet [4]. The long electrodes (belt type) were placed under the shoulder and the lumber region of an adult, while reference electrode was placed under hip and thighs. A conducting tail in each of the electrode was tailored beneath the bed sheet to convey the ECG signal to the pre-amp module. The measurement was taken place inside a RFshielded room with constant temperature of $22^{\circ} \mathrm{C}$. Cotton 
was used as main dielectric material in subject clothing and bed sheet as well.

An interim study of capacitive ECG measurement in a clinical practice was conducted by Eilebrecht et al at Aachen university clinic in 2009 [5]. They integrated stiff capacitive electrodes into a pillow to use it in a clinical bed or a chair with active driven reference electrode, analogous to "Driven right leg circuit".

After successfully integrating textile capacitive ECG electrodes into Automotive environment [6][7], in the following we propose a textile electrode based, non-contact ECG monitoring system integrated in hospital items like a stretcher, a standard hospital bed and a manual wheel chair.

\section{CAPACITIVE SENSING OF BODY PARAMETERS}

\section{A. Capacitive Coupling}

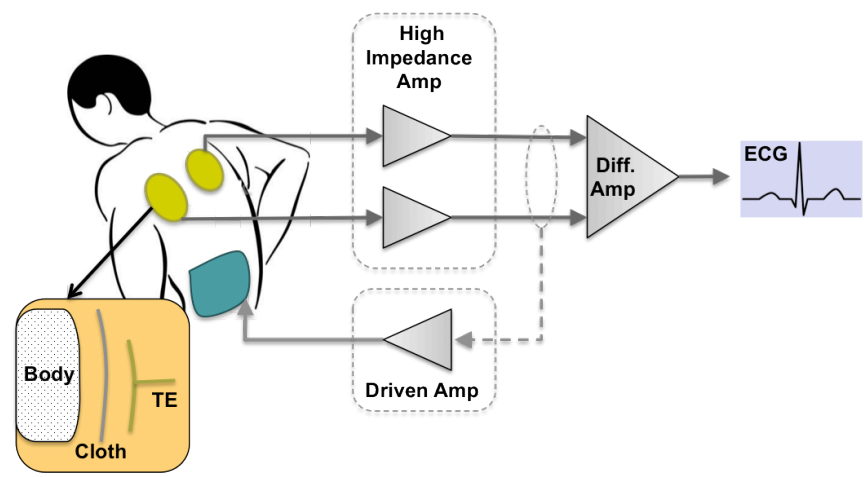

Figure 1. Sketch of a Capacitively Coupled Electrocardiography system. The insert shows a magnification of a capacitive contact with dielectric material between skin and electrode.

Potential generated by the heart is conducted through the tissue to be measurable at the epidermis (outer skin layer). Equipotential lines form the potential across the whole body create a body surface potential mapping [8]. Conventional Electrocardiography uses a galvanic contact with the skin to acquire these potentials [9]. However, the body surface can be considered as conductive plate and if there is any conductive surface close to the body separated by an electrical insulation, they will form a capacitor $\left(\mathrm{C}_{\mathrm{el}}\right)$ according to equation (1) [10]. Here clothes, air and/or any material between the two surfaces act as dielectric material of the capacitor as shown in the Fig.1 (insert).

$$
C_{e l}=\mathcal{E}_{r} \mathcal{E}_{0} \frac{A}{d}
$$

Fig. 1 shows a capacitive ECG system configuration. The body and the capacitive electrode (TE) form the capacitor $\left(\mathrm{C}_{\mathrm{el}}\right)$ to couple the potential from the body to the high impedance amplifier [10]. The input amplifier works as a buffer to transfer the signal from a high impedance source to its low impedance output for the next stage of signal processing. Signals from the buffers are fed to differential amplifiers to minimize common mode signals and to amplify the differential signal.

Further improvement in the common mode cancellation can be achieved by a driven seat circuit. The circuit uses the unity gain signals from the buffer to feed it back to the body through a driving amplifier to the driven seat electrode after adding and amplifying the signals [11][12].

\section{B. High input impedance pre amplifier}

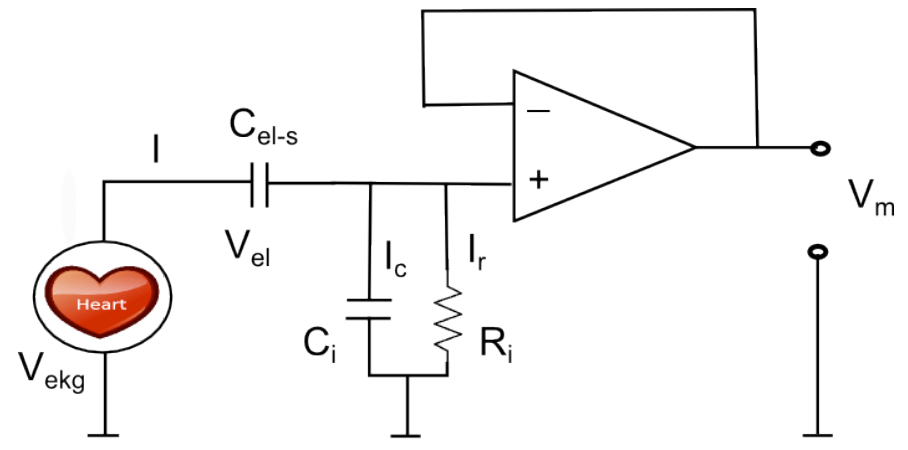

Figure 2. Input stage of the CCECG System

The capacitance formed by a body and the capacitive electrode is very low (approx 1-100 pF) [13] for various distances, hence resulting in contact impedances in the range of Giga Ohms. The typical amplitude of an ECG signal is in the range of 1 to $2 \mathrm{mV}$. While getting the ECG Signal through capacitively coupled measurement reduces the amplitude further. So in this case, it is very crucial to have proper impedance matching to achieve the signal with minimum loses.

$$
\begin{aligned}
& V_{e k g}=V_{e l}+V_{m} \\
& V_{e k g}=\frac{1}{C_{e l-s}} \int I d t+V_{m} \\
& V_{e k g}=\frac{1}{C_{e l-s}} \int\left(I+I_{r}\right) d t+V_{m} \\
& V_{e k g}=\frac{1}{C_{e l-s}} \int\left(C_{i} \frac{d V_{m}}{d t}+\frac{V_{m}}{R_{i}}\right) d t+V_{m} \\
& V_{e k g}=\frac{C_{i}}{C_{e l-s}} V_{m}+\frac{1}{R_{i}} \int \frac{V_{m}}{C_{e l-s}} d t+V_{m} \\
& V_{m}=V_{e k g}-\frac{C_{i}}{C_{e l-s}} V_{m}+\frac{1}{R_{i}} \int \frac{V_{m}}{C_{e l-s}} d t
\end{aligned}
$$


The input stage of the capacitive coupling is depicted in the Fig. 2. According to the Kirchhoff's law, potential distribution in the loop would be as follows.

In the equation (2), $\mathrm{C}_{\mathrm{el}-\mathrm{s}}, \mathrm{R}_{\mathrm{i}}$, and $\mathrm{C}_{\mathrm{i}}$ represent $\mathrm{C}_{\mathrm{el}}$ plus series capacitor if any, total input resistance and total input capacitance respectively.

Here from the equation (2), it can be deduced that two conditions would be necessary to minimize the signal attenuation. Followings are the two conditions (3) and (4), where $\mathrm{X}_{\mathrm{Cel-s}}$ is the impedance of the capacitance $\mathrm{C}_{\mathrm{el-s}}$.

$$
\begin{aligned}
& \mathrm{C}_{\text {el-s }} \gg \mathrm{C}_{\mathrm{i}} \\
& \mathrm{Ri} \gg \mathrm{X}_{\text {Cel-s }}
\end{aligned}
$$

This means lesser input capacitance of the amplifier than the skin-electrode capacitance and higher input impedance of the amplifier than the skin-contact impedance betters the signal coupling and reduces signal attenuation.

\section{SYSTEM COMPONENTS}

\section{A. Capacitive-coupled Electrode}

After trying stiff capacitor plates in earlier work [14], a textile electrode was designed. The electrodes were printed and developed with help of TITV, Greiz, Germany. The electrode contains 3 conductive (silver printed) and 3 isolating textile layers (PES-Knitted substrate-7058, Thorey). The silver gel to print the electrodes were made up of $75 \%$ silver solvated in 2-butoxyethanol. The structure of the electrode can be seen in the Fig. 3. Various layers are highlighted with different colors, Sensing layer (Sin, red), Guarding layer (GR, blue), Grounding layer (GN, Green).
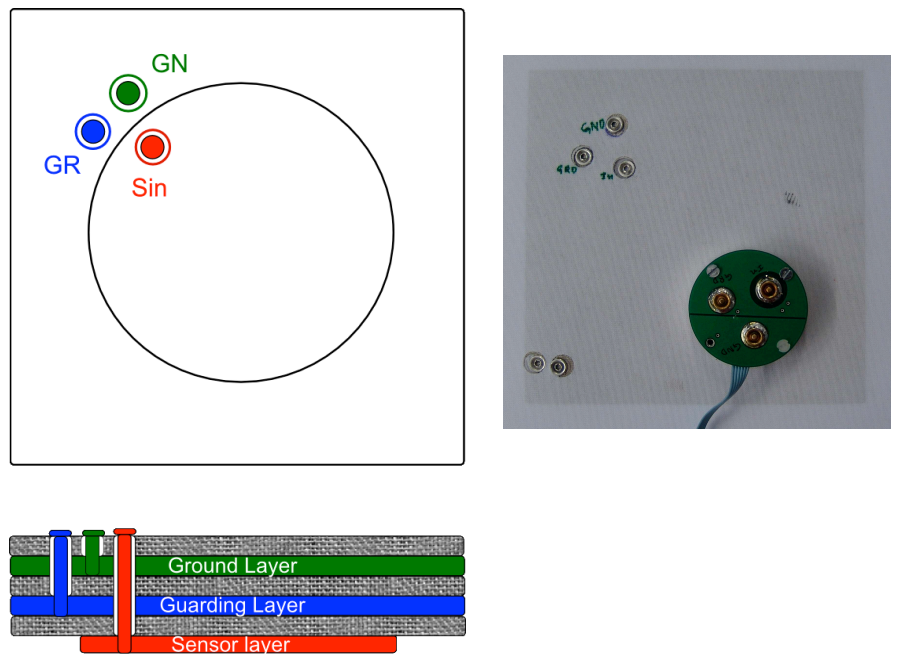

Figure 3. The textile electrode structure: front view (top-left), side view (left-bottom) and rear view of the implemented electrode (top-right).

Guarding layer and grounding layer work as active and passive shield for the sensor layer. Guarding is a technique to actively shield a surface against any parasitic effect (e.g. coupling with surrounding noises) by driving the shield (GR) with common mode voltage [15]. This same surrounding potential will avoid any coupling with any potential nearby, hence helps to keep the high input impedance of the amplifier intact.

Grounding the layer on top of the guarding layer further shields the input [16]. Each layer of the electrode is connected with the preamplifier through a conductive snap fastener as seen in the implemented electrode (Fig. 3).

\section{B. Preamplifier}

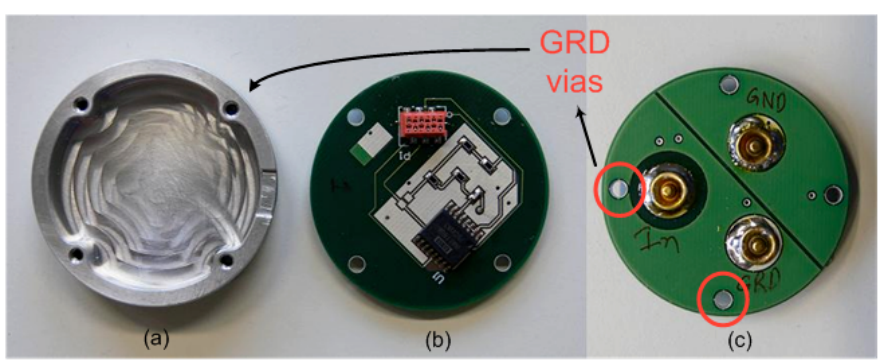

Figure 4. Pre-amplifier module: module housing (a), top view of the electronics circuit (b), snap connection to the electrode (c).

A high input impedance amplifier with biasing was needed to couple the ECG signal as explained in the section II.B. An ultra low input bias current instrumentation amplifier INA116 (Burr Brown Corporation, USA) has been identified for this application. It has very high input resistance $(1015 \Omega)$ and the very low input capacitance $(0.2$ $\mathrm{pF}$ ) [17], thus nicely coping with the requirements. A modular design containing preamplifier (INA116) PCB and an actively shielded aluminum case are shown in the Fig. 4.

A four layer printed circuit board (PCB) was used to shield the input signal from the textile electrode through conductive snap fasteners. Guarding and grounding layer were also implemented in the PCB on top of the bottom layer (connecting layer) as shown in the figure. These shielding layers help to minimize parasitic effect as described in the section III. A.

Two holes (left and bottom) seen in the Fig. 4 (c) are built as conducting vias to connect to the aluminum housing. This connection added to the guarding plane on the top layer of the PCB (silver colored) ultimately joins guarding pins on the INA116; so does the guarding layer of the textile electrode. Driving the housing with the common mode voltage guards the input by avoiding coupling of surroundings as mentioned earlier.

\section{Signal conditioning}

A capacitive ECG system is highly prone to noise due to its ultra high and sometimes fluctuating input impedance. In this case, signal processing plays a very important role to remove noise.

Capacitive coupled signal from each of the CCECG electrodes is fed to a differential filter in the analog signal processing toolbox (90IPB, Frequency devices). The differential signal from the two CCECG electrodes is filtered 
with a window of $1-37 \mathrm{~Hz}$ by an $8^{\text {th }}$ order elliptical (1.56) band-pass filter. The signal was amplified before and after the filtering by gain of 10 and 20 respectively.

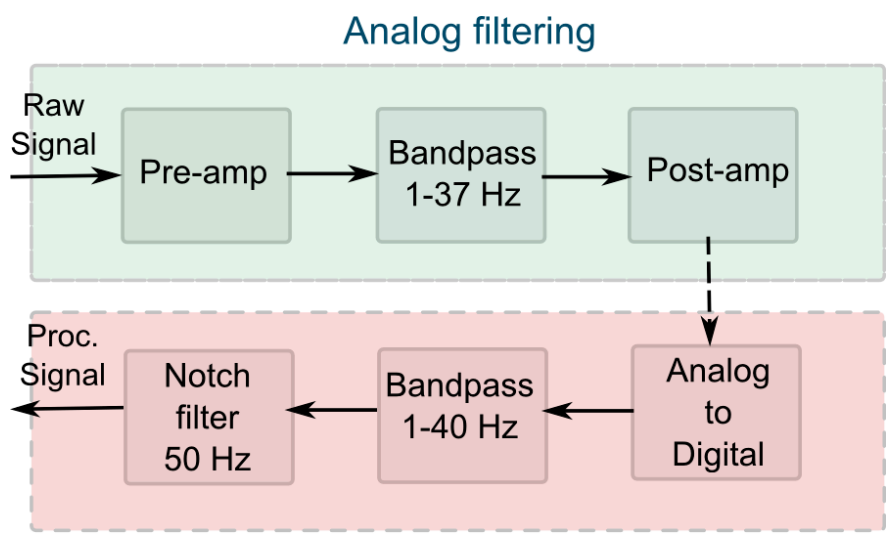

Digital filtering

Figure 5. Block diagram of the signal processing.

The filtered signal is acquired by a LabVIEW program for further processing after digitizing it with a DAQ card (NI U-9162). Here the signal is digitally filtered by a $4^{\text {th }}$ order Butterworth band-pass filter with a bandwidth of 1-40 Hz and a $4^{\text {th }}$ order Butterworth notch filter with $50 \mathrm{~Hz}$. The resulting signal is displayed by the LabVIEW application.

\section{CCECG SET UP IN A CLINICAL ENVIRONMENT}

As mentioned earlier, to meet the objective of CCECG in a clinical environment, we setup the system on three objects frequently used by patients: a stretcher, a bed and a wheelchair. Objects were chosen such that the patient's ECG can be monitored non-obtrusively from the moment, he enters into the hospital until he leaves it. In order to validate and compare ECG signals detected by CCECG system textile electrodes (TITV-Greiz, Germany) were also used for detecting 2-lead contact ECG signal.

\section{A. Stretcher}

A stretcher often is a patient's first-point of contact with the clinical environment. For us it is the first contact with the patient's back surface and hence furnishes a good platform to capture contactless ECG in ambulatory conditions. As shown in Fig. 6 (left), CCECG electrodes are simply placed in the thoracic section of the stretcher and are facing towards the back of the patient. No gelled electrodes have to be fixed on the patient. A squared driven electrode is located at the lumbar section of the stretcher. For validation purposes contact ECG electrodes made of conductive textile are attached on the stretcher's handrest and are easily reachable by a volunteer's hands.

\section{B. Hospital Bed}

After the patient is admitted to the hospital, he commonly spends major portion of his hospitalized time in a clinical bed. Fig. 6 (middle) shows the locations of two CCECG electrodes on the bed-sheet. The contact ECG electrodes are also placed on two sides of the bed-sheet and easily accessible by the volunteers' hands.

\section{Wheel Chair}

During several phases of their hospitalized recovery patients are allowed to mobilize themselves on wheelchairs. Wheelchairs also provide firm contacts between the patient's back and the wheelchair's back rest. On the other hand, a rigid contact of patient's bottom within the seat facilitates a good driven electrode. Our exemplary wheelchair was prepared for CCECG measurement by placing electrodes in the back-rest and the driven electrode on the seat. The contact ECG electrodes were stuck onto both arm-rests.

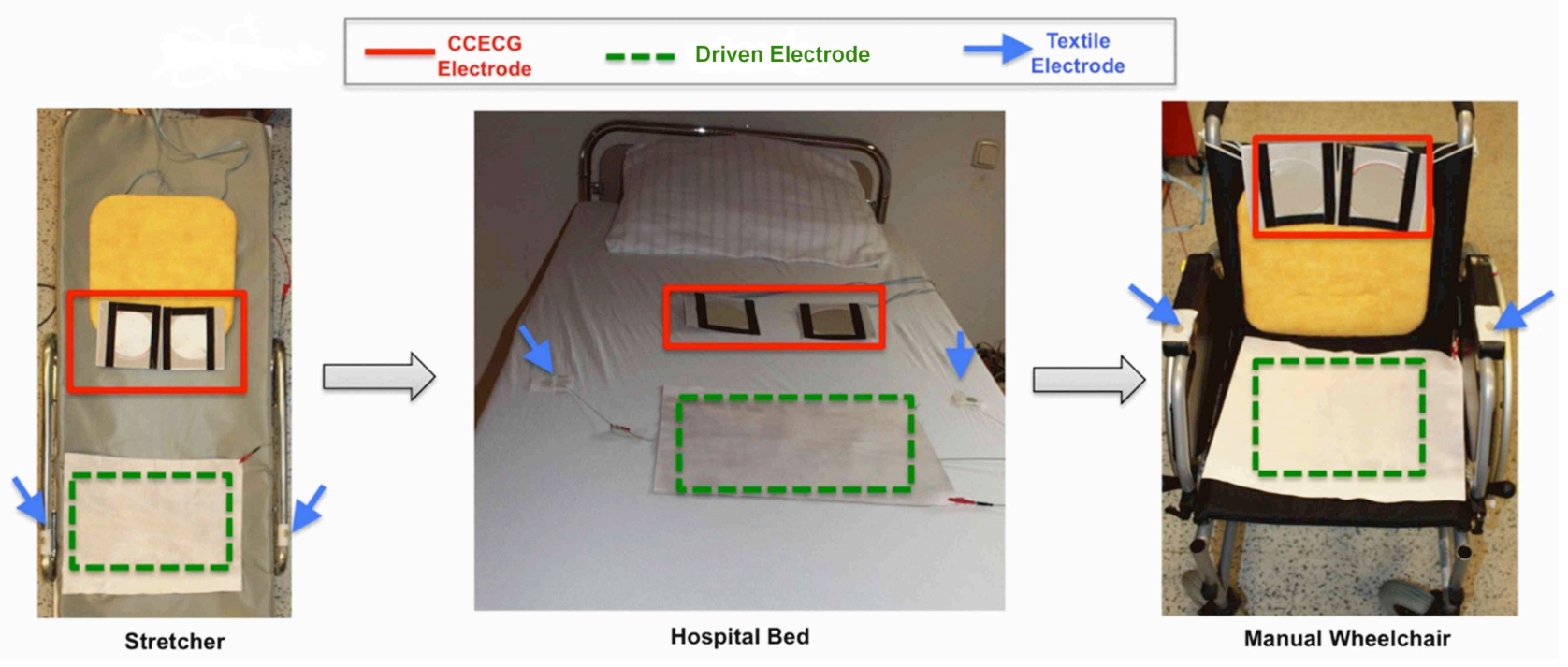

Figure 6. Experimental setup for various clinical environments. 


\section{EXPERIMENTAL RESULTS \& DISCUSSION}

A standard CCECG system setup was applied to all three cases. The only difference was the placement of the textile electrodes as seen in the experimental setups. A minimum of three volunteers occupied each test setup for up to 30 minutes. The measurements were taken placed in a hospital for clinical bed setup and in an electronics laboratory for the wheel chair and stretcher setup to have it in realistic situations.

To demonstrate long term feasibility of Capacitive Coupled Electrocardiography, 5 second and 60 second signal snippets are presented here for each of the cases. A conventional II lead ECG was used to compare it to the CCECG signal. Conventional and capacitive electrocardiograms are plotted in all of the graphs with green and red respectively.

\section{A. $\quad$ Stretcher}

Volunteers were asked to take the conventional supine position on the stretcher, as illustrated earlier. In this position electrodes were located posterior of the subject's heart. Test subjects wore a t-shirt (cotton, $0.4 \mathrm{~mm}$ thick) with a sweater (cotton, $1.23 \mathrm{~mm}$ thick) on top and corduroy trousers (cotton, $1.6 \mathrm{~mm})$.

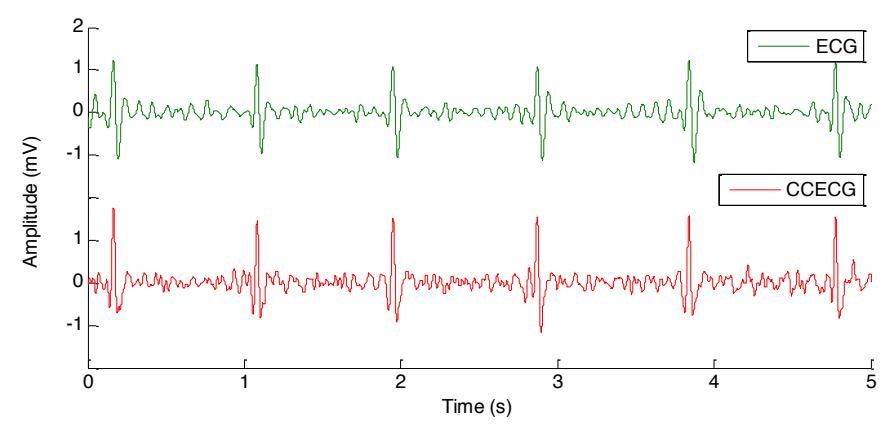

Figure 7. ECG measurement on the stretcher for 5 seconds.

Fig. 7 depicts exemplary capacitive ECG recordings together with the contact ECG. Resemblance of the capacitive ECG is evident when compared to the contact ECG. A minute long signal is shown in Fig. 8 for consideration of heart rate (HR) and heart rate variability (HRV) analysis. Movement artifacts appear in this signal as larger spikes than the ECG signal itself.

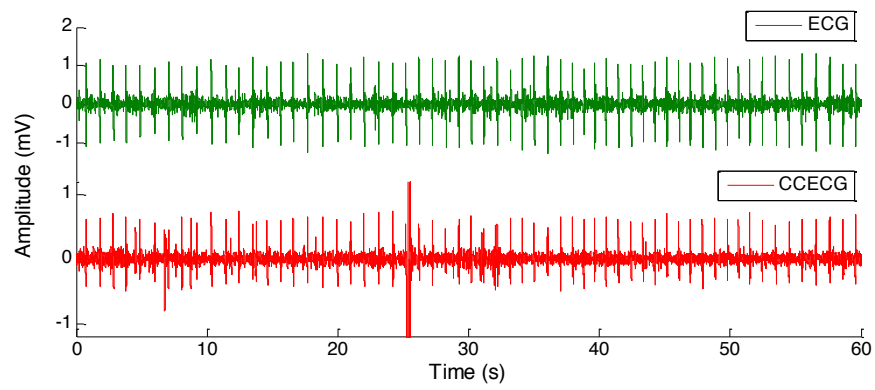

Figure 8. ECG measurement on the stretcher for a minute.

\section{B. Hospital Bed}

Here, volunteers lied down in supine position emulating a patient on clinical bed. Placement of the CCECG electrodes was little separated and right behind heart of the subject. The softness and sponginess of the clinical bed-mattresses is an advantage in maintaining an adequate contact pressure between the subjects's back and the mattress surface. The exemplary subject was a 28 year old male, weighed $68 \mathrm{~kg}$ and was $178 \mathrm{~cm}$ tall. The subject wore a t-shirt (cotton, $0.35 \mathrm{~mm}$ thick) and jeans trousers (cotton, $.87 \mathrm{~mm}$ ).

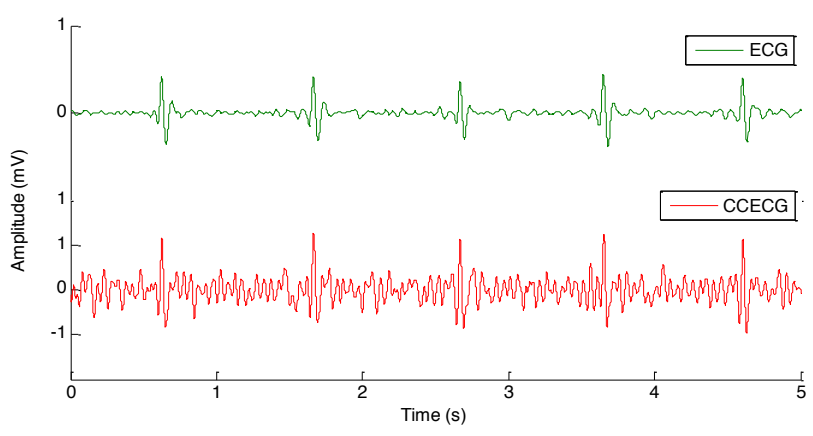

Figure 9. ECG measurement on the Hospital bed for 5 seconds.

An ECG signal from this setup shows more interferences as depicted in the Fig. 9. The signal displays a noisy baseline and hence a worse signal to noise ration than compared to the stretcher setup. Baseline noise as well as some other artifacts including movement artifacts are visible in a longer signal (Fig. 10).

This clearly needs to be addressed by appropriate signal processing.

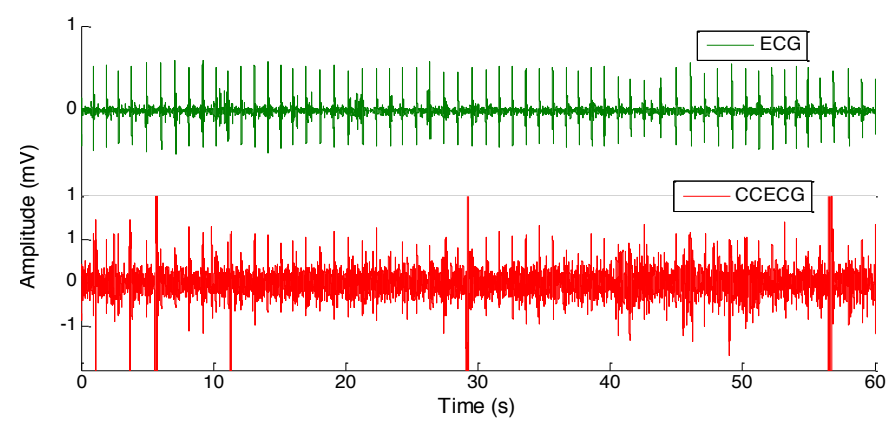

Figure 10. ECG measurement on the hospital bed for a minute.

\section{Wheel Chair}

In this case, the volunteer was a 34 year old female, weighing $67 \mathrm{~kg}$ at $167 \mathrm{~cm}$ height. The subject wore a t-shirt (viscous, $0.34 \mathrm{~mm}$ thick) and trousers (polyamide \& cotton, $0.96 \mathrm{~mm}$ ). The subject was asked to sit in the wheel chair comfortably and as relaxed as possible while holding the textile contacts for conventional ECG measurement. 


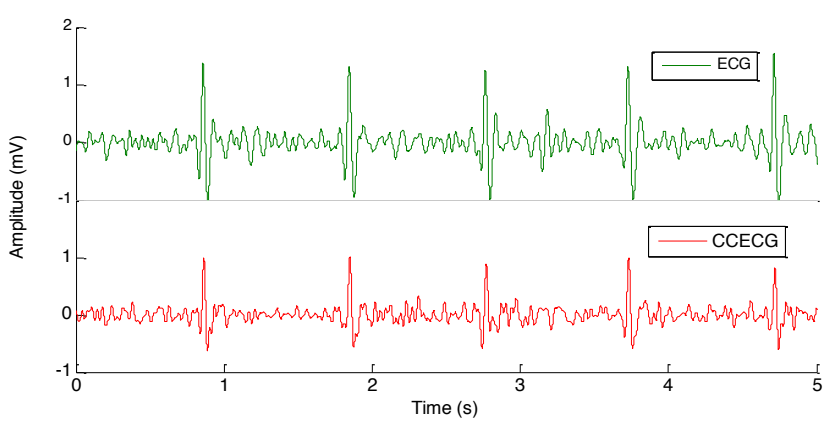

Figure 11. ECG measurement in the wheel chair for 5 seconds.

Measurement in this setup is presented in Fig. 11. A long term measurement in Fig. 12 also shows ECG like peaks in addition to few artifactitious spikes.

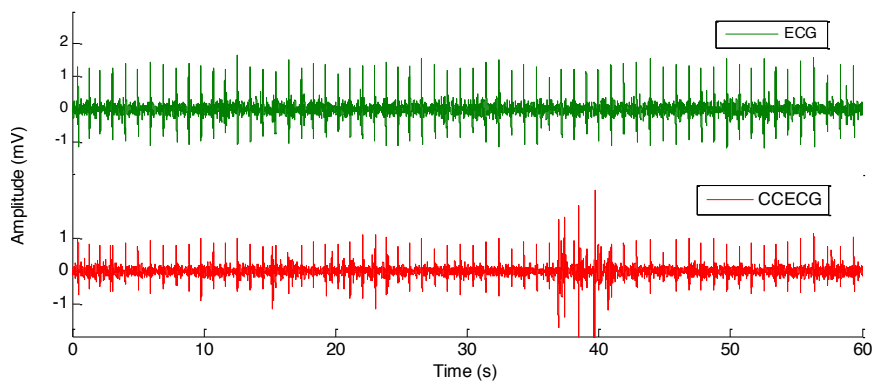

Figure 12. ECG measurement in the wheelchair for a minute.

\section{CONCLUSIONS \& OUTLOOK}

With the ageing society the demand for permanent health monitoring will grow, no the least due to the growing number of persons living in nursing homes or serviced apartments. Together with the presumed advantages of continuous ECG monitoring in the clinical environment, a need for contact-free, non-obtrusive ECG recordings arises.

With this study, we showed the feasibility of non-contact ECG measurements by a lab demonstrator of a capacitive coupled ECG system. Our system uses conductive textiles actively shielded by a driven seat circuit. Flexible textile electrodes were placed on three very common clinical fitments. Here the demonstrators were able to successfully record ECG signals from volunteers without the need to glue conductive electrodes on the subject's skin. Signals are of sufficient quality as to use them for further analysis like heart rate (HR) or heart rate variability (HRV), however they are, due to their delicate recording physics, prone to interference and movement artifacts.

In order to utilize non-contact ECG measurements in locations of high nursing demand further steps to improve stability, signal quality and long term acceptance have to be taken.

Still, we interpret our results as motivating enough to envision the potential future care facility featuring a personal, non-obtrusive and yet permanent health monitoring system providing reassuring baseline data and fast response times against serious health problems.

\section{ACKNOWLEDGMENT}

The authors would like to thank the BMBF (German ministry for education and research) for funding the work of the INSITEX project.

\section{REFERENCES}

[1] Richardson, P. C., “The Insulated Electrode," In Proceedings of the 20th Annual Conference on Engineering in Medicine and Biology. Boston, MA (USA), 1967, p. 157.

[2] Y. G. Lim, K. K. Kim and K. S. Park, "ECG recording on a Bed During Sleep Without Direct Kin-Contact," IEEE Tras. On Bio. Engg., vol. 54, no. 4, pp. 718-725, April 2007.

[3] A. Ueno et al, "Capacitive sensing of Electrocardiographic potential through cloth from the dorsal surface of the body in a supine position: A prelminary study," IEEE Trans. on Biomed. Engg., Vol. 54, No.4, pp. 759-766, April 2007.

[4] K. Wu and Y. Zhang, "Contactless and Continuous Monitoring of Heart Electric Activities through Clothes on a Sleeping Bed," Proc.of $5^{\text {th }}$ Internt. Conf. on IT and Appl in Biomedicine, pp. 282- 285, 2005.

[5] B. Eilebrecht et al, "Implementation of a capacitive ECG measurment system in clinical practice: an interim report," IFMBE Proc. 25, pp. 370-372, 2009.

[6] B. Chamadiya, M. Wagner, W. Wondrak and U. Hofmann, "Active passenger safety by intelligent textile," $7^{\text {th }}$ internt. Conf. on Wearable micro and nano Tech. for personalized Healthcare, Berlin, Germany, 2010.

[7] B. Chamadiya, S. Heuer M. Wagner and U. Hofmann, "Textile Capacitive Electrocardiography for Automotive environment," BIODEVICES 2011, Interntl. Conf. on Biomedical electronics and devices, Rome, Italy, pp. 422-425, January 2011.

[8] J. Malmivuo and R. Plonsey, Bioelectromagnetism, principles and applications of bioelectric and biomagnetic fields. New York : Oxford University Press.1995, pp.16-19.

[9] J. D. Bronzino, The Biomedical Engineering Handbook. Florida, CRC Press LLC. 2000, pp. 181-190.

[10] A. J. Clippingdale, R. J. Prance, T.D. Clark, and C. Watkins, "Ultrahigh impedance capacitively coupled heart imaging array," Rev. Sci. Instrum. 65, pp. 269-270, January 1994.

[11] K. Kim, Y. Lim, and K. S. Park, "Common mode noise cancellation for electrically non-contact ECG measurement system on a chair," Proc. 2005 IEEE Engg in Med. \& Bio. 27 ${ }^{\text {th }}$ annual conference, Shanghai, China, September 2005.

[12] K. Lee, S. Lee, K. Sim, K. Kim and K. S. Park, "Noise reduction for non-contact electrocardiogram measurement in daily life," Computers in Cardiology 2009, Vol. 36, pp. 493-496.

[13] R. J. Prance, A Debray, et al, "An ultra-low-noise electrical-potential probe for human-body scanning," Meas. Sci. Technol. 11, pp. 291297, January 2000.

[14] B. K. Chamadiya, S. Heuer, U. G. Hofmann and M. Wagner, "Towards a capacitively coupled electrocardiography system for car seat integration," IFMBE Proceedings 22, ECIFMBE 2008, pp. 12171221, September 2008.

[15] R. Pallas-Areny and J. G. Webster, Analog Signal Processing. Newyork : Wiley-Interscience Publication. 1999, pp. 482-484.

[16] A. Rich, "Shielding and Guarding, how to exclude interference-type noise, what to do and why to do - A rationale approach," Analog Dialogue 17-1, pp. 124-129, 1983.

[17] Burr-Brown corporation, "INA 116, an ultra low input bias current instrumentation amplifier," Datasheet, pp. 1-9, September 2010. 